\title{
Aspectos da microbiota vaginal e a relação com a candidíase em mulheres gestantes: uma revisão de literatura
}

Aspects of vaginal microbiota and the relationship with candidiasis in pregnant women: a literature review

Aspectos de la microbiota vaginal y la relación con candidiasis en mujeres embarazadas: revisión de la Literatura

Roberto de Faria Espinheiro ORCID: https://orcid.org/0000-0002-6786-1708 Faculdade de Medicina Estácio de Castanhal, Brasil

E-mail: robertofespinheiro@gmail.com

Maria Clara Coelho Monteiro

ORCID: https://orcid.org/0000-0002-1437-0104 Faculdade de Medicina Estácio de Castanhal, Brasil

E-mail: mariaclaracmonteiro76@gmail.com

Rafael Hipolito Pires Batista

ORCID: https://orcid.org/0000-0001-9043-482X Faculdade de Medicina Estácio de Castanhal, Brasil

E-mail: rafaelbatistabiomedicina@gmail.com

Manoela Paulo de Oliveira Miléo Gomes

ORCID: https://orcid.org/0000-0003-1063-6267

Faculdade de Medicina Estácio de Castanhal, Brasil

E-mail: manoelapm.med@gmail.com

Rebeca Elise de Lima Pantoja

ORCID: https://orcid.org/0000-0002-7578-612X

Faculdade de Medicina Estácio de Castanhal, Brasil E-mail: becaelise@gmail.com

Sofia Alessandra Naiff Araújo

ORCID: https://orcid.org/0000-0003-3090-0731

Faculdade de Medicina Estácio de Castanhal, Brasil

E-mail: sofiaaraujo985@gmail.com

Paulo Afonso Oliveira Gomes

ORCID: https://orcid.org/0000-0001-5578-6854

Faculdade de Medicina Estácio de Castanhal, Brasil

E-mail: pauloafonsoliveirag@gmail.com

Max Chaves Monta Junior

ORCID: https://orcid.org/0000-0002-4644-4913

Faculdade de Medicina Estácio de Castanhal, Brasil

E-mail: maxmotajr@gmail.com

Milena Souza Amaral

ORCID: https://orcid.org/0000-0003-0949-1742

Faculdade de Medicina Estácio de Castanhal, Brasil

E-mail: milenasouzaamarall@gmail.com

Adonis de Melo Lima

ORCID: https://orcid.org/0000-0003-1334-1106

Faculdade de Medicina Estácio de Castanhal, Brasil

E-mail: adonislima@gmail.com

Luiz Mário Pará Rodrigues

ORCID: https://orcid.org/0000-0002-1479-9568

Faculdade de Medicina Estácio de Castanhal, Brasil E-mail: Lmpara@gmail.com

Clebson Pantoja Pimentel

ORCID: https://orcid.org/0000-0002-0497-5927

Faculdade de Medicina Estácio de Castanhal, Brasil

E-mail: clebsonpp@yahoo.com.br

Silvan Francisco da Silva

ORCID: https://orcid.org/0000-0002-9597-5788

Faculdade de Medicina Estácio de Castanhal, Brasil E-mail: sfdasilva@gmail.com 


\author{
Darlen Cardoso de Carvalho \\ ORCID: https://orcid.org/0000-0002-2716-860X \\ Faculdade de Medicina Estácio de Castanhal, Brasil \\ E-mail: darlen.c.carvalho@gmail.com \\ Maurício Ferreira Gomes \\ ORCID: https://orcid.org/0000-0002-8943-4272 \\ Faculdade de Medicina Estácio de Castanhal, Brasil \\ E-mail: mauricioneuroquimica@gmail.com
}

\begin{abstract}
Resumo
A microbiota vaginal possui papel importante no equilíbrio, na manutenção e prevenção da saúde vaginal das mulheres, possuindo vasta quantidade de microrganismos. Candida spp. é uma das espécies de fungos que mais causa infecções nos seres humanos. Durante a gravidez, onde os níveis hormonais estão elevados e ocorre variação do $\mathrm{pH}$ vaginal, esses microrganismos podem colonizar a vagina e consequentemente propiciar uma infecção. O presente trabalho visa avaliar os aspectos da microbiota vaginal e sua correlação com a candidíase vulvovaginal na gravidez, e suas formas de diagnóstico. O presente estudo caracteriza-se por ser uma revisão integrativa de literatura, onde foram utilizadas as plataformas científicas PubMed, Google Acadêmico, Medline e Scielo Artigos como fonte de dados. Os dados gerados na pesquisa demonstraram a importância da compreensão sobre a microbiota vaginal. Além de evidenciar a importância de estudos a respeito da candidíase vulvovaginal em gestantes, e como outras espécies de Candida sp. estão afetando e elevando os índices de pacientes com corrimento vaginal, além da Candida albicans, a qual é a espécie mais estudada desse gênero. Também observar a relevância do diagnóstico dessa enfermidade. Concluímos assim que o conhecimento dos aspectos clínicos da sintomalogia, principalmente, durante a gravidez é uma importante ferramenta para o diagnóstico precoce durante o período pré-natal. A transmissão vertical se mostra presente nas infecções em recémnascido. A espécie mais evidente desse gênero é a C. albicans contudo, as espécies de Candida não albicans estão cada vez mais presentes nas infecções causadas pelo gênero Candida spp.
\end{abstract}

Palavras-chave: Microbiota; Candidíase; Gravidez; Diagnóstico.

\begin{abstract}
The vaginal microbiota has an important role in the balance, maintenance and prevention of vaginal health in women, having a vast amount of microorganisms. Candida spp. it is one of the fungal species that most causes infections in humans. During pregnancy, when hormone levels are high and there is a variation in the vaginal $\mathrm{pH}$, these microorganisms can colonize the vagina and consequently lead to an infection. This study aims to evaluate aspects of the vaginal microbiota and its correlation with vulvovaginal candidiasis in pregnancy, and its forms of diagnosis. The present study is characterized by being an integrative literature review, where the scientific platforms PubMed, Academic Google, Medline and Scielo Articles were used as data sources. The data generated in the research demonstrated the importance of understanding the vaginal microbiota. In addition to highlighting the importance of studies on vulvovaginal candidiasis in pregnant women, and how other Candida sp. are affecting and increasing the rates of patients with vaginal discharge, in addition to Candida albicans, which is the most studied species of this genus. Also note the relevance of the diagnosis of this disease. Thus, we conclude that knowledge of the clinical aspects of symptoms, especially during pregnancy, is an important tool for early diagnosis during the prenatal period. Vertical transmission is present in newborn infections. The most evident species of this genus is C. albicans, however, nonalbicans Candida species are increasingly present in infections caused by the genus Candida spp.
\end{abstract}

Keywords: Microbiota; Candidiasis; Pregnancy; Diagnosis.

\title{
Resumen
}

La microbiota vaginal tiene un papel importante en el equilibrio, mantenimiento y prevención de la salud vaginal en la mujer, al contar con una gran cantidad de microorganismos. Candida spp. es una de las especies de hongos que más causa infecciones en humanos. Durante el embarazo, cuando los niveles hormonales son altos y hay una variación en el $\mathrm{pH}$ vaginal, estos microorganismos pueden colonizar la vagina y, en consecuencia, provocar una infección. Este estudio tiene como objetivo evaluar aspectos de la microbiota vaginal y su correlación con la candidiasis vulvovaginal en el embarazo y sus formas de diagnóstico. El presente estudio se caracteriza por ser una revisión integradora de la literatura, donde se utilizaron como fuentes de datos las plataformas científicas PubMed, Academic Google, Medline y Scielo Articles. Los datos generados en la investigación demostraron la importancia de comprender la microbiota vaginal. Además de resaltar la importancia de los estudios sobre candidiasis vulvovaginal en mujeres embarazadas, y cómo otras Candida sp. están afectando y aumentando las tasas de pacientes con flujo vaginal, además de Candida albicans, que es la especie más estudiada de este género. También tenga en cuenta la relevancia del diagnóstico de esta enfermedad. Así, concluimos que el conocimiento de los aspectos clínicos de los síntomas, especialmente durante el embarazo, es una herramienta importante para el diagnóstico precoz durante el período prenatal. La transmisión vertical está presente en las infecciones del recién nacido. La especie más evidente de este género es C. albicans, sin embargo, las especies de Candida no albicans están cada vez más presentes en infecciones causadas por el género Candida spp.

Palabras clave: Microbiota; Candidiasis; Embarazo; Diagnóstico. 


\section{Introdução}

A microbiota vaginal possui uma formação variável e dinâmica de fungos, peptococcos, bactérias aeróbicas, bactérias anaeróbicas facultativas comensais e lactobaciluus que possuem papel fundamental na manutenção e preservação da saúde. O desequilíbrio do ecossistema vaginal pode ocorrer por uma multiplicidade de fatores como, função do ciclo de vida, fases do ciclo menstrual, gravidez, infecções e comportamentos sexuais. Além disso, o uso de antibióticos, higiene vaginal, contraceptivos, terapia hormonal, mudanças no $\mathrm{pH}$ e até hábitos alimentares podem contribuir para esse descontrole (Oliveira \& Carneiro, 2020).

A candidíase é uma doença causada por leveduras do gênero Candida spp. (Vieira \& Santos, 2017). Essa doença afeta milhões de mulheres todos os anos e tem sido considerada um importante problema de saúde pública (Gonçalves et al., 2016). As espécies pertencentes a esse gênero são a segunda causa mais frequente de infecções fúngicas no mundo e apresentam considerável variação filogenética e fenotípica (Whibley \& Gaffen, 2015). As espécies mais comuns que causam vulvovaginites são Candida albicans, Candida. glabrata, Candida tropicalis, Candida parapsilosis e Candida krusei (Gonçalves et al., 2016).

A morfologia de Candida spp. é predominantemente em forma de levedura, entretanto, algumas espécies apresentam dimorfismo, podendo crescer em forma de pseudo-hifas ou hifas (Whibley \& Gaffen, 2015). A forma hifal tem um papel importante em causar doenças ao invadir células epiteliais e causar danos aos tecidos (Sudbery, 2011). A identificação do tipo específico de levedura é importante para se instituir o tratamento e evitar resistências, além de incluir parceiro na terapêutica (Souza et al., 2012).

O conhecimento a respeito da imunidade à infecção por Candida spp. são quase sempre baseados no que se sabe sobre a imunidade a C. albicans. Contudo, existem diferenças importantes na imunidade às espécies de Candida sp., as espécies de Candida não albicans (CNA) surgem como patógenos emergentes (Whibley \& Gaffen, 2015). Há tempos, acreditava-se que as espécies de Candida spp. participavam passivamente no processo de infecção fúngica oportunista, contudo, hoje, sabe-se que essas leveduras participam ativamente no estabelecimento da infecção, a patogenicidade relacionada aos fatores de virulência: adesão, formação de biofilme, produção de enzima hidrolítica extracelular, formação de hifas e troca fenotípica (Gonçalves et al., 2016).

O aumento dos níveis de resistência das espécies de Candidas não albicans aos tratamentos comumente utilizados associados à crescente identificação dessas espécies em mulheres com a candidíase vulvovaginal (CVV) destacam a importância da identificação de espécies de Candida spp. em amostras vaginais, isso permite mais informações aos profissionais de saúde visando um tratamento mais adequado as pacientes (Gonçalves et al., 2016).

O gênero Candida spp. é de grande importância médica, responsável pela alta incidência de vulvovaginites em mulheres gestantes. Essas leveduras fazem parte da microbiota natural do ser humano, estabelecendo uma relação de comensalismo, contudo, podem ser patógenos oportunistas quando ocorre uma ruptura da microbiota ou por comprometimento da eficiência do sistema imune (Lima \& Cohen, 2017).

O corrimento vaginal provocado pela infecção por Candida spp. interfere diretamente na vida da gravida, sendo um dos sintomas que motiva a consulta ginecológica (Lima \& Cohen, 2017). Pacientes relatam elevados níveis de incomodo, principalmente decorrente do prurido, muitas vezes o diagnóstico é realizado de forma empírica, levando a prescrição precipitada de medicamentos ou à automedicação. Sendo assim, de suma importância a identificação das leveduras através da análise de suas características micromorfológicas e perfil bioquímico (Soares et al., 2018).

Durante a gestação, os níveis hormonais de estrogênio e progesterona podem favorecer a colonização vaginal por microrganismos, dentre eles incluindo Candida spp., e podem ou não está associados a complicações obstétricas e infecções neonatais (Freitas et al., 2020). A CVV materna é um importante fator de risco para colonização por Candida sp. e infecções dos 
lactantes, sendo a transmissão vertical um ponto importante no desenvolvimento na infecção do recém-nascido, sendo necessário a realização de bom pré-natal, para diagnóstico precoce dessa enfermidade (Zisova et al, 2016).

Dessa forma, podemos destacar a importância do conhecimento sobre a CVV em grávidas e sua relevância no período pré-natal e para os recém-nascidos. Com isso, este trabalho teve como objetivo realizar uma revisão atualizada da literatura a respeito da microbiota vaginal, bem como, a importância do conhecimento da candidíase vulvovaginal em gestantes, além de evidenciar as principais formas de diagnóstico dessa enfermidade e a importância do acompanhamento clínico.

\section{Metodologia}

Foi aplicado um método específico de revisão que sumariza estudos concluídos anteriormente - Revisão Integrativa de Literatura - para prover um entendimento compreensivo sobre a problemática específica ou um problema de saúde. Essa revisão integrativa tem o potencial de construir um conhecimento sistêmico a partir de conteúdos pré-revisados e filtrados quanto a relevância dentro da problemática.

Com o método manuseado, foi gerado um potencial de caracterizar os fatores moduladores da microbiota vaginal e a sua relação com a candidíase na gestação e seu índice de incidência. Esta pesquisa envolve cinco etapas: identificação da problemática discutida, busca e levantamento de literaturas científicas, análise de dados relevantes, filtragem baseada em critérios de inclusão ou exclusão e redação de conclusão.

Foram utilizados os seguintes descritores para a busca de artigos nas plataformas científicas, sendo a busca realizada pelos autores de forma independente, utilizando a combinação dos seguintes descritores nos idiomas inglês e português: "microbiota", "candidíase", "gravidez" e "diagnóstico". A plataformas utilizadas como bases de dados para o levantamento desses artigos foram: PubMed, Google Acadêmico, Medical Literature Analysis and Retrieval Sistem online (Medline) e Scientific Electronic Library Online (Scielo).

\subsection{Critérios de Inclusão e Exclusão}

Os critérios de inclusão foram artigos de grande valor científico, que abordam assuntos referentes aos descritores selecionados, discutindo a influência do diagnóstico da candidíase durante a gravidez, trabalhos que tratavam conceitualmente fatores que modulam a microbiota vaginal, todos publicados nos últimos dez anos (2011-2021). Foram excluídos os textos que não englobaram dentro dos critérios anteriormente mencionados. 
Figura 1. Fluxograma descritivo da seleção dos artigos do estudo.

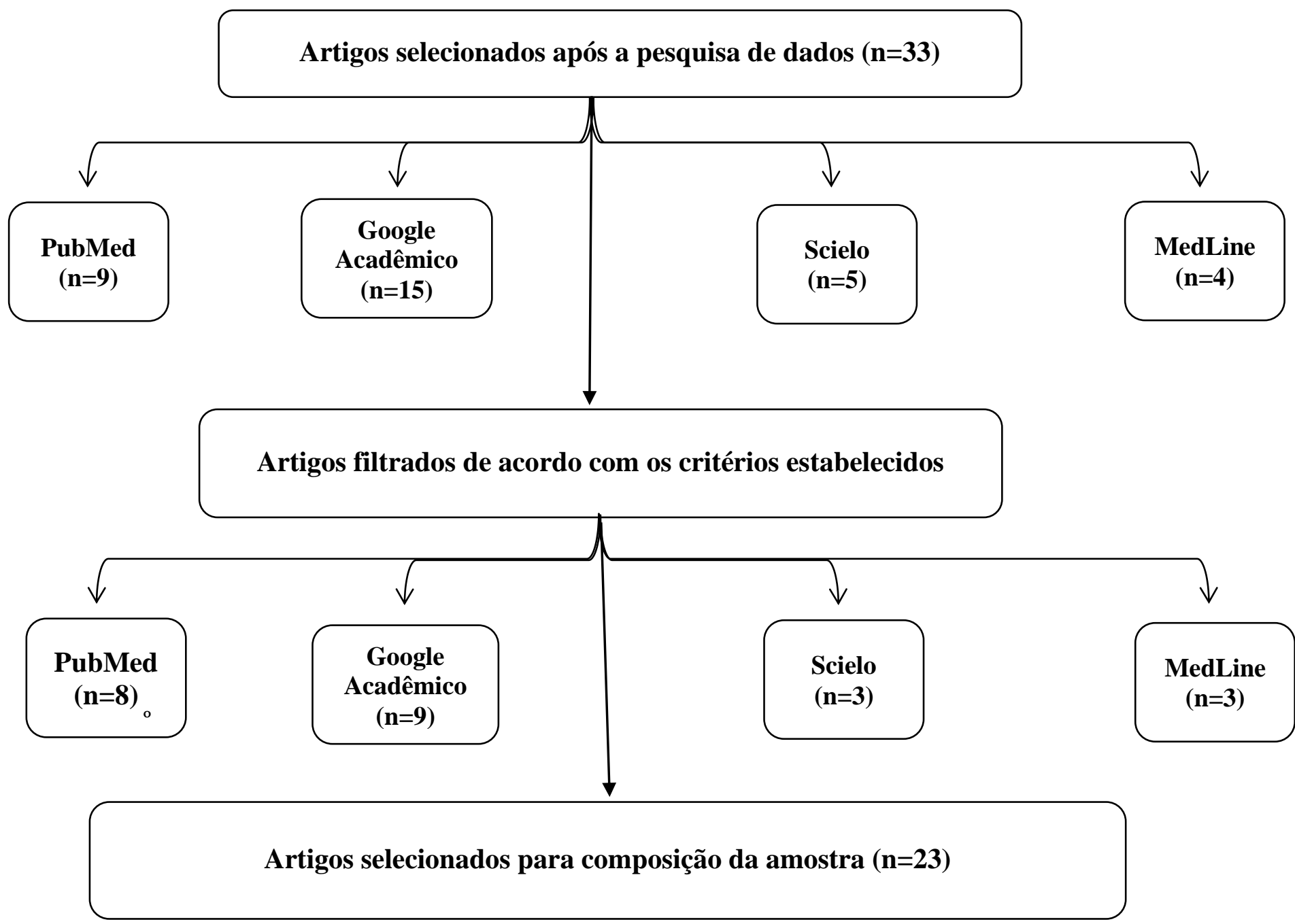

Fonte: Autores (2021).

\section{Resultados e Discussão}

Os dados obtidos no presente estudo por meio de uma revisão de literatura científica permitiram analisar os aspectos da microbiota vaginal e sua relação com a candidíase durante a gravidez. Diante disso, a busca foi realizada por meio de bases de dados utilizando diversas combinações com os seguintes descritores: "microbiota", "candidíase", "gravidez" e "diagnóstico". Dessa forma, as combinações tiveram um total de 33 artigos e foram selecionados apenas 23 artigos. O Quadro 1 aborda os artigos que foram selecionados para auxiliar na construção do trabalho.

Quadro 1. Levantamento dos estudos sobre os aspectos associados a microbiota vaginal e a candidíase na gestação.

\begin{tabular}{|c|c|l|c|c|c|}
\hline $\mathbf{N}^{\mathbf{0}}$ & Autor /Ano & \multicolumn{1}{|c|}{ Título } & Objetivos & Metodologia & Resultados \\
\hline $\mathbf{0 1}$ & $\begin{array}{c}\text { Almeida } \text { et al., } \\
2017\end{array}$ & $\begin{array}{l}\text { Efeito da suplementação de } \\
\text { Lactobacillus spp no } \\
\text { tratamento e prevenção de } \\
\text { Candidíase vulvovaginal e } \\
\text { vaginose bacteriana }\end{array}$ & $\begin{array}{c}\text { Avaliar os benefícios do uso } \\
\text { dos lactobacillus spp. no } \\
\text { tratamento das } \\
\text { vulvovaginites }\end{array}$ & $\begin{array}{c}\text { Revisão bibliográfica: } \\
\text { análise de dados }\end{array}$ & $\begin{array}{l}\text { O uso de lactobacillus } \\
\text { apresentam melhoras no } \\
\text { tratamento de infecções }\end{array}$ \\
\hline $\mathbf{0 2}$ & $\begin{array}{c}\text { Bernardo \& Lima, } \\
2015\end{array}$ & $\begin{array}{l}\text { Ocorrência de Candidíase no } \\
\text { exame citológico de pacientes } \\
\text { do Hospital Geral de Curitiba }\end{array}$ & Diagnosticar a CVV & $\begin{array}{c}\text { Papanicolaou de } \\
\text { rotina }\end{array}$ & $\begin{array}{l}\text { Total de amostras: } 511 ; \\
\text { Candida } \\
(4,10 \%)\end{array}$ \\
spp.: \\
21
\end{tabular}




\begin{tabular}{|c|c|c|c|c|c|}
\hline $\mathbf{0 3}$ & Dias et al, 2011 & $\begin{array}{l}\text { Vulvovaginal candidiasis in } \\
\text { Mato Grosso, Brazil: } \\
\text { Pregnancy status, causative } \\
\text { species and drugs tests }\end{array}$ & $\begin{array}{l}\text { Investigar as espécies } \\
\text { antifúngicas de leveduras }\end{array}$ & $\begin{array}{l}\text { Cultura de corrimento } \\
\text { vaginal sugestivo de } \\
\text { candidíase }\end{array}$ & $\begin{array}{l}\text { 93\% de candidíase em } \\
\text { gestantes }\end{array}$ \\
\hline 04 & Duarte et al, 2019 & $\begin{array}{l}\text { Métodos diagnósticos para a } \\
\text { caracterização de candidíase e } \\
\text { papiloma vírus humano }\end{array}$ & $\begin{array}{l}\text { Identificar as técnicas de } \\
\text { diagnóstico para candidíase }\end{array}$ & $\begin{array}{c}\text { Detalhar o } \\
\text { funcionamento dos } \\
\text { testes que auxiliam no } \\
\text { diagnóstico de } \\
\text { Candidíase }\end{array}$ & $\begin{array}{l}\text { As técnicas auxiliam no } \\
\text { processo de tratamento }\end{array}$ \\
\hline 05 & $\begin{array}{l}\text { Freitas et al., } \\
\quad 2020\end{array}$ & $\begin{array}{l}\text { Prevalência de } \\
\text { microrganismos em secreção } \\
\text { vaginal de gestantes de alto } \\
\text { risco de uma maternidade em } \\
\text { Caruaru, Pernambuco, Brasil }\end{array}$ & $\begin{array}{l}\text { Determinar a prevalência de } \\
\text { microrganismos em } \\
\text { gestantes de alto risco }\end{array}$ & $\begin{array}{l}\text { Amostras de secreção } \\
\text { vaginal foram } \\
\text { submetidas a } \\
\text { coloração de Gram e } \\
\text { culturas. (92 } \\
\text { gestantes) }\end{array}$ & $\begin{array}{l}\text { Candida spp. Foi a mais } \\
\text { prevalente }(31,52 \% \text { das } \\
\text { amostras })\end{array}$ \\
\hline 06 & $\begin{array}{l}\text { Ghaddar } \text { et al. } \\
2020\end{array}$ & $\begin{array}{l}\text { Prevalence and antifungal } \\
\text { susceptibility of } \begin{array}{r}\text { Candida } \\
\text { albicans causing } \\
\text { discharge among } \\
\text { women in Lebanon }\end{array}\end{array}$ & $\begin{array}{c}\text { Determinar a prevalência de } \\
\text { candida em gestantes } \\
\text { sintomáticas }\end{array}$ & $\begin{array}{l}\text { Foram selecionadas } \\
258 \text { mulheres } \\
\text { grávidas com } \\
\text { corrimento vaginal e } \\
\text { foram submetidas a } \\
\text { cultura } \\
\end{array}$ & $\begin{array}{l}39 \% \text { foram positivos } \\
\text { para Candida sp.; } 42 \% \\
\text { C. albicans; } 41 \% \text { C. } \\
\text { glabrata; } 17 \% \text { krusei }\end{array}$ \\
\hline 07 & $\begin{array}{l}\text { Gonçalves et al. } \\
2016\end{array}$ & $\begin{array}{l}\text { Vulvovaginal candidiasis: } \\
\text { Epidemiology, microbiology } \\
\text { and risk factors }\end{array}$ & $\begin{array}{l}\text { Reconhecer os fatores de } \\
\text { risco para CVV }\end{array}$ & Revisão de Literatura & $\begin{array}{l}\text { Fatores relacionados ao } \\
\text { hospedeiro e fatores } \\
\text { comportamentais: } \\
\text { gravidez, reposição } \\
\text { hormonal, prática sexual } \\
\text { e uso de } \\
\text { anticoncepcionais }\end{array}$ \\
\hline 08 & $\begin{array}{l}\text { Lima \& Cohen, } \\
2017\end{array}$ & $\begin{array}{l}\text { Candidíase vulvovaginal } \\
\text { recorrente em gestantes }\end{array}$ & $\begin{array}{l}\text { Descrever a candidíase de } \\
\text { repetição em gestantes }\end{array}$ & Revisão de Literatura & $\begin{array}{l}\text { Consequência do risco } \\
\text { potencial ao feto e a } \\
\text { saúde materna }\end{array}$ \\
\hline 09 & $\begin{array}{l}\text { Menezes } \text { et al., } \\
\quad 2012\end{array}$ & $\begin{array}{l}\text { Identificação molecular e } \\
\text { suscetibilidade antifúngica de } \\
\text { Candida parapsilosis isoladas } \\
\text { no Ceará, Brasil }\end{array}$ & $\begin{array}{c}\text { Identificar a } \\
\text { susceptibilidade de } C . \\
\text { parapsilosis aos } \\
\text { antifúngicos }\end{array}$ & $\begin{array}{l}\text { Testes fenotípicos e } \\
\text { moleculares }\end{array}$ & $\begin{array}{lr}\text { As sepas de } C \text {. } \\
\text { parapsilosis } \\
\text { mostraram sensíveis aos } \\
\text { antifúngicos testados }\end{array}$ \\
\hline 10 & Mucci et al., 2017 & $\begin{array}{l}\begin{array}{l}\text { Prevalence of } \begin{array}{r}\text { Candida } \\
\text { albicans, } \\
\text { Candida }\end{array} \\
\text { dubliniensis and Candida } \\
\text { africana in pregnant women } \\
\text { suffering from vulvovaginal } \\
\text { candidiasis in Argentina }\end{array} \\
\end{array}$ & $\begin{array}{c}\text { Avaliar a prevalência de } \\
\text { Candida sp. }\end{array}$ & $\begin{array}{l}\text { Testes fenotípicos e } \\
\text { moleculares }\end{array}$ & $\begin{array}{l}\text { Candida albicans foi a } \\
\text { espécie predominante } \\
\text { em } 42(80,7 \%) \text { dos } 52 \\
\text { isolados. }\end{array}$ \\
\hline 11 & $\begin{array}{c}\text { Oliveira \& } \\
\text { Carneiro, } 2020\end{array}$ & $\begin{array}{l}\text { Fatores associados a } \\
\text { alterações da microbiota no } \\
\text { trato genital feminino inferior }\end{array}$ & $\begin{array}{c}\text { Avaliar fatores associados } \\
\text { as alterações da microbiota } \\
\text { vaginal }\end{array}$ & Revisão de literatura & $\begin{array}{l}\text { Fatores predominantes: } \\
\text { relações sexuais } \\
\text { frequentes, } \\
\text { medicamentos } \\
\text { sabonetes íntimos } \\
\end{array}$ \\
\hline 12 & $\begin{array}{l}\text { Peixoto et al., } \\
2014\end{array}$ & $\begin{array}{l}\text { Candidíase - uma revisão de } \\
\text { literatura }\end{array}$ & $\begin{array}{l}\text { Estudar a diversidade do } \\
\text { gênero Candida }\end{array}$ & Revisão de literatura & $\begin{array}{lr}\text { Destaca-se } & a \\
\text { importância } & \text { dessa } \\
\text { patologia e r sua } \\
\text { associação com fatores } \\
\text { locais e sistêmicos } \\
\text { predisponentes }\end{array}$ \\
\hline 13 & $\begin{array}{l}\text { Rodrigues et al., } \\
2013\end{array}$ & 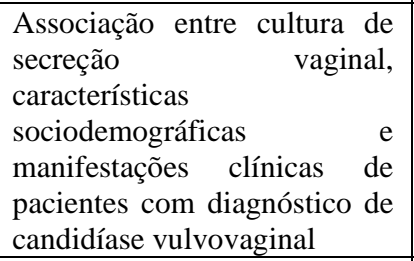 & $\begin{array}{c}\text { Investigar o perfil } \\
\text { epidemiológico de pacientes } \\
\text { com CVV }\end{array}$ & $\begin{array}{l}\text { Coleta de dados e } \\
\text { teste molecular }\end{array}$ & $\begin{array}{l}\text { Foram avaliadas } 69 \\
\text { pacientes, } \\
\text { predominando mulheres } \\
\text { casadas }(56,5 \%) \text {, vida } \\
\text { sexual ativa }(97,1 \%) \text {, } \\
\text { gestantes }(34,8 \%)\end{array}$ \\
\hline 14 & $\begin{array}{l}\text { Sangaré et al., } \\
2017\end{array}$ & $\begin{array}{l}\text { Prevalence of vulvovaginal } \\
\text { candidiasis in pregnancy at } \\
\text { three health centers in Burkina } \\
\text { Faso }\end{array}$ & $\begin{array}{l}\text { Determinar a prevalência de } \\
\text { Candida spp. em mulheres } \\
\text { grávidas }\end{array}$ & Coleta de amostras & $\begin{array}{l}\text { A prevalência de } \\
\text { vulvovaginites } \\
\text { mulheres grávidas foi } \\
\text { relativamente superior } \\
\text { nesse estudo }\end{array}$ \\
\hline 15 & Soares et al., 2018 & $\begin{array}{l}\text { Candidíase vulvovaginal: uma } \\
\text { revisão de literatura com }\end{array}$ & $\begin{array}{l}\text { Estudar a diversidade do } \\
\text { gênero Candida }\end{array}$ & Revisão de literatura & $\begin{array}{l}\text { CVV não é uma doença } \\
\text { letal, no entanto, os }\end{array}$ \\
\hline
\end{tabular}




\begin{tabular}{|c|c|c|c|c|c|}
\hline & & $\begin{array}{l}\text { abordagem para Candida } \\
\text { albicans }\end{array}$ & & & $\begin{array}{lr}\begin{array}{l}\text { sinais } \begin{array}{r}\text { sintomas } \\
\text { causados } \\
\text { estão associados a uma }\end{array} \\
\text { morbidade significativa }\end{array} \\
\end{array}$ \\
\hline 16 & Sudbery, 2011 & $\begin{array}{l}\text { Growth of Candida albicans } \\
\text { hyphae }\end{array}$ & $\begin{array}{c}\text { Descrever a compreensão } \\
\text { de processos moleculares } \\
\text { no crescimento polarizado } \\
\text { de hifas }\end{array}$ & Testes moleculares & $\begin{array}{l}\text { Poucos ou nenhuma das } \\
\text { funções dos genes } \\
\text { identificados sugerem } \\
\text { um envolvimento óbvio } \\
\text { com o crescimento } \\
\text { polarizado }\end{array}$ \\
\hline 17 & $\begin{array}{l}\text { Tansarli et al., } \\
2016\end{array}$ & $\begin{array}{l}\text { Abnormal vaginal flora in } \\
\text { symptomatic non-pregnant } \\
\text { and pregnant women in a } \\
\text { Greek hospital: a prospective } \\
\text { study }\end{array}$ & $\begin{array}{c}\text { Avaliar a flora vaginal } \\
\text { anormal }\end{array}$ & $\begin{array}{c}\text { Teste de } 761 \text { mulheres } \\
\text { sintomáticas }\end{array}$ & 579 com candidíase \\
\hline 18 & Tapia et al., 2016 & $\begin{array}{l}\text { Genotyping and Persistence of } \\
\text { Candida albicans from } \\
\text { Pregnant Women with } \\
\text { Vulvovaginal Candidiasis }\end{array}$ & $\begin{array}{l}\text { Estudar genótipos de } \\
\text { Candida albicans }\end{array}$ & $\begin{array}{l}\text { Swabs vaginais, } 30 \\
\text { suspeitas e } 33 \\
\text { controles }\end{array}$ & $\begin{array}{l}30 \% \text { grupo controle, } \\
80 \% \text { do grupo suspeitos } \\
\text { foram positivos }\end{array}$ \\
\hline 19 & $\begin{array}{l}\text { Vasconcelos et } \\
\text { al., } 2016\end{array}$ & $\begin{array}{l}\text { Estudo comparativo entre } \\
\text { terapia oral e local no } \\
\text { tratamento de corrimentos } \\
\text { vaginais: } \\
\text { tricomoníase e Candidíase, } \\
\text { bacteriana }\end{array}$ & $\begin{array}{c}\text { Comparar a eficácia de } \\
\text { tratamentos }\end{array}$ & Usando RAPD & $\begin{array}{l}\text { Não existe comprovação } \\
\text { de superioridade entre } \\
\text { medicamentos orais ou } \\
\text { locais }\end{array}$ \\
\hline 20 & $\begin{array}{c}\text { Vieira \& Santos, } \\
2017\end{array}$ & $\begin{array}{l}\text { Mecanismos de resistência de } \\
\text { Candida albicans aos } \\
\text { antifúngicos anfotericina B, } \\
\text { fluconazol e caspofungina }\end{array}$ & $\begin{array}{c}\text { Estudar os mecanismos de } \\
\text { resistência da Candida } \\
\text { albicans }\end{array}$ & Revisão de literatura & $\begin{array}{lr}\text { Descrição } & \text { dos } \\
\text { mecanismos } & \text { de } \\
\text { resistência da } & \text { Candida } \\
\text { albicans } & \end{array}$ \\
\hline 21 & $\begin{array}{l}\text { Whibley \& } \\
\text { Gaffen, } 2015\end{array}$ & $\begin{array}{l}\text { Beyond Candida albicans: } \\
\text { Mechanisms of immunity to } \\
\text { non-albicans Candida species }\end{array}$ & $\begin{array}{l}\text { Analisar estudos de } \\
\text { candidas não albicans }\end{array}$ & Revisão de literatura & $\begin{array}{l}\text { A importância de } \\
\text { estudar casos de } \\
\text { candidas não albicans }\end{array}$ \\
\hline 22 & $\begin{array}{c}\text { Zimmermmann et } \\
\text { al., } 2019\end{array}$ & $\begin{array}{l}\text { Frequência e etiologia de } \\
\text { fluxos genitais na gravidez }\end{array}$ & $\begin{array}{c}\text { Avaliar a frequência de } \\
\text { fluxos genitais patológicos } \\
\text { na gravidez }\end{array}$ & Coleta de dados & $\begin{array}{l}\text { Foi identificado em } 127 \\
(34,7 \%) \text { pacientes com } \\
\text { fluxo genital patológico, } \\
\text { no qual } 67 \quad(18,3 \%) \\
\text { foram para candidíase }\end{array}$ \\
\hline 23 & $\begin{array}{l}\text { Zisova et al., } \\
2016\end{array}$ & $\begin{array}{l}\text { Vulvovaginal Candidiasis in } \\
\text { Pregnant Women and its } \\
\text { Importance for Candida } \\
\text { Colonization of Newborns }\end{array}$ & $\begin{array}{c}\text { Determinar a incidência da } \\
\text { candidíase vulvovaginal em } \\
\text { gestantes }\end{array}$ & $\begin{array}{c}\text { Amostras de } \\
\text { secreções vaginais } \\
\text { suspeitas de } \\
\text { candidíase }\end{array}$ & $\begin{array}{l}28,75 \% \text { das gestantes e } \\
22,22 \% \text { dos recém- } \\
\text { nascidos }\end{array}$ \\
\hline
\end{tabular}

Fonte: Autores (2021).

A microbiota vaginal tem papel fundamental na manutenção e preservação da saúde. Diante disso, a microbiota vaginal normal possui microrganismos que se colonizam e vivem em equilíbrio na vagina feminina. Como mencionado anteriormente, a microbiota vaginal é composta por fungos, peptococos, bactérias aeróbicas e anaeróbicas facultativas comensais, e os lactobacilos que possui grande importância por manter o equilíbrio do pH da vagina (Oliveira \& Carneiro, 2020).

A alteração da microbiota normal da vagina pode levar ao aparecimento de patologias e infecções como, vaginose bacteriana, candidíase vulvovaginal, vaginite aeróbica, infecção por clamídia, gonorreia e Infecções Sexualmente Transmissíveis - IST's (Duarte et al., 2019).

Os fatores que alteram ou modulam a microbiota vaginal seriam, função do ciclo de vida, fases do ciclo menstrual, infecções, atividade sexual, número de parceiros sexuais pela alta exposição a diversos tipos de microrganismos, imunidade, idade por haver alterações hormonais desde o nascimento até a vida adulta, hábitos alimentares, e a localização anatômica do trato genital feminino podem levar as alterações microbiológicas da microbiota vaginal das mulheres (Oliveira \& Carneiro, 2020). 
A gestação é um período marcado por alterações hormonais nas mulheres, levando a altos níveis de estrogênio e progesterona facilitando a colonização de microrganismos patogênicos na microbiota vaginal (Zimmermmann et al., 2019). Além disso, a diabetes, a imunossupressão, o uso de antibióticos, quimioterápicos, radioterápicos e outros medicamentos serão fatores que contribuirão para que a mulher seja ainda mais vulnerável a essas infecções (Freitas et al., 2020). Outrossim, o uso de roupas íntimas apertadas ou sintéticas, e de produtos de higiene íntima, lubrificantes, óleos, perfumes e duchas vaginais podem fazer uma limpeza mecânica excessiva das bactérias comensais que são importantes para o equilíbrio da microbiota vaginal alterando o $\mathrm{pH}$ vaginal e desenvolver até irritações na pele. Métodos contraceptivos, como o DIU poderá contribuir para susceptibilidade de algumas infeções por levar ao ressecamento da região vaginal, por conta disso, as mulheres que fazem uso do DIU devem ter um acompanhamento médico regular. A alteração da microbiota vaginal saudável é gerada por uma série de fatores e multiplicidades comportamentais, fisiológicas, patológicas e medicamentosas que desencadeiam de forma direta ou indireta uma modificação no equilíbrio da microbiota vaginal das mulheres (Oliveira \& Carneiro, 2020).

Dessa forma, as perspectivas terapêuticas para essas condições seriam as mulheres realizarem o preventivo regularmente, educação sexual, uso de preservativo nas relações sexuais, melhor hábito de higiene íntima, controle da diabetes, uso racional de antibióticos, maior atenção à saúde pública para mulheres gestantes, imunossupremidas, em quimioterapia, e em menopausa pois, são as mais propensas a ter um desequilíbrio da microbiota vaginal. Seguindo todos os cuidados necessários as mulheres poderão ter uma diminuição das patologias, uma melhoria na qualidade de vida e no seu bem-estar (Duarte et al., 2019).

Anomalias hormonais na flora vaginal como inflamações foram diagnosticadas em algumas mulheres gestantes e não gestantes, causada por alterações hormonais durante a gravidez e período pré-menstrual (Tapia et al., 2016). As proporções das inflamações foram similares, tendo nos dois casos, diagnósticos de infecção por Candida sp. e outras inflamações recorrentes de alterações hormonais da microbiota vaginal (Tansarli et al., 2016). As alterações hormonais observadas na gravidez provocam aumento do glicogênio vaginal, o que reduz o pH local e favorece a proliferação fúngica, facilitando para que ocorra a candidíase, tornado uma infecção bastante frequente durante a gestação (Vasconcelos et al., 2016).

Espécies do gênero Candida sp. podem causar infecções oportunistas, contudo, subsistem em relação simbiótica com a microbiota vaginal, assim a colonização assintomática pode persistir por anos (Ghaddar et al., 2020). As espécies de Candida são uma das principais causas de infecções fúngicas, com destaque para Candida albicans, contudo, espécies de Candidas nãoalbicans apresentam implicações importante no tratamento da enfermidade (Whibley \& Gaffen, 2015). O conhecimento da estrutura etiológica atual de VVC em mulheres grávidas e a prevalência de espécies de Candida sp. determina a abordagem terapêutica subsequente mais apropriada (Zisova et al., 2016).

A CVV materna é um importante fator de risco para colonização por Candida sp. e infecções dos lactantes, sendo a transmissão vertical um ponto importante no desenvolvimento na infecção do recém-nascido, sendo necessário a realização de bom pré-natal, para diagnóstico precoce dessa enfermidade (Zisova et al., 2016). As gestantes com CVV sintomáticas devem ser tratadas em qualquer momento da gestação para evitar parto prematuro (Souza et al., 2012).

Os sintomas mais frequentes em pacientes com CVV são: corrimento vaginal, coceira vulvovaginal, sensação de queimação vulvovaginal, irritação vaginal e secreção vaginal com odor fétido (Mucci et al., 2017; Sangaré et al., 2017). Essas manifestações clínicas, caso não tratadas, podem ir de uma infecção localizada de mucosas até uma doença disseminada potencialmente fatal, isso é influenciado diretamente pela resposta imunológica do paciente, que determinará o tipo e extensão da infecção causada pela Candida sp (Peixoto et al., 2014). Situações como diminuição do pH vaginal, principalmente no período pré-menstrual e situações de debilidade do hospedeiro favorecem o aparecimento da CVV, além de outros fatores como uso de antibióticos de amplo espectro de forma irracional, uso de corticoides e hábitos de higiene inadequados (Almeida et al., 2017; bernardo \& lima, 2015). 
A transmissão vertical tem papel importante na colonização neonatal por Candida sp. nos primeiros dias de vida e essa descoberta facilita o prognóstico da infecção por Candida albicans no neonato, diminuindo os riscos de complicações pós-natais (Zisova et al., 2016).

A espécie $C$. albicans é a mais frequente causando CVV em gestantes, em pacientes na Argentina esteve presente em $80 \%$ das pacientes sintomáticas, em 5,7 \% dos casos estava associada também a cândidas não albicans (Mucci et al., 2017). Em Burkina Faso, foram encontrados Candida sp. em 22,71\% dos 229 esfregaços vaginais analisados, nos quais, os isolados foram maiores em pacientes sintomáticas, do total de 52 cepas encontradas a C. albicans foi a mais prevalente 40,39\%, sendo também encontrado uma grande quantidade de candidas não-albicans: C. glabrata (32,69\%), C. tropicalis (15,38\%) e C. krusei (11,54\%), além disso, é possível destacar que $C$. glabrata foi mais prevalente em pacientes assintomáticos enquanto que a prevalência de C. albicans foi alta em pacientes sintomáticas (Sangaré et al., 2017). No Líbano o crescente número de cepas de CNA em mulheres grávidas aparece como novas e preocupantes (Ghaddar et al., 2020).

$\mathrm{Na}$ Bulgária, de 80 mulheres grávidas saudáveis que apresentavam suspeitas de candidíase foram submetidas a exames de cultura, obtendo 28,75\% de Candida spp., sendo C. albicans a espécie mais frequente, nos isolados, os recém-nascidos (RN) apresentaram 22,22\% de positividade, e todos os isolados dos RN as cepas eram idênticas as das mães (Zisova et al., 2016). Desta forma, fica evidente a importância da transmissão vertical e os problemas que podem surgir nos RN. No estado de Pernambuco-Brasil foram estudadas 92 pacientes gravidas, foi encontrado uma prevalência de 31,52\% de Candida spp., dentre elas 10,35\% apresentavam diabetes (Freitas et al., 2020).

A Candidíase vulvovaginal (CVV) tem se apresentado como um diagnóstico constante em ginecologia, caracterizando um aumento em sua incidência. Clinicamente, a paciente apresenta intenso prurido vulvar, leucorreia, dispareunia, disúria, edema e eritema vulvovaginal, sendo o prurido analisado o sintoma mais recorrente quando a CVV é comparada a vulvovaginites de outra etiologia (Almeida et al., 2017), o prurido vulvar pode se apresentar de uma forma acentuada , que o ato constante de coçar, produz escoriações e até fissuras superficiais fazendo com que essas lesões se espalhem pelo períneo, região perianal e inguinal (Soares et al., 2018).

O primeiro diagnóstico da candidíase vulvovaginal se dá por um exame pélvico, os sintomas clínicos incluem pruridos na região da vulva, secreções brancas e espessas, edemas e eritemas nos tecidos da área vulvar e vaginal. Além do olhar clínico deve-se observar as características da secreção vaginal, avaliando critérios como, cor, viscosidade e presença ou não de odores. Exames laboratoriais com especificidade na sintomatologia devem ser realizados, coletando e avaliando a amostra da secreção ao microscópio e observar se de fato se trata de uma infecção apenas por um tipo de microrganismo (Duarte et al., 2019).

O diagnóstico determinado por identificação das leveduras presentes nas secreções vaginais ocorre por métodos rápidos, como o exame a fresco e coloração de Gram, que são analisados microscopicamente (Soares et al., 2018) os isolamentos primários de leveduras de amostras clínicas são realizados em meio ágar Sabouraud glucose ou ágar batata dextrose (Menezes et al., 2012). Através do rigor na análise de sinais e sintomas durante o diagnóstico clínico, relacionado com às boas práticas laboratoriais e carga microbiana presente na secreção vaginal, observa se um resultado positivo na correlação entre diagnóstico de CVV e isolamento de leveduras (Rodrigues, 2013).

No exame a fresco o material é retirado das paredes laterais da vagina, com espátula de Ayre ou swab, que é então depositado em lâmina e misturado com uma ou duas gotas de solução fisiológica e coberta com lamínula. A adição de uma ou duas gotas de $\mathrm{KOH}$ a $10 \%$ na secreção vaginal destrói os elementos celulares, facilitando a visualização das leveduras e pseudohifas (Soares et al., 2018).

O papel de pH Whatman pode ser utilizado para identificação da candidíase, sendo um método de análise físico-química de baixo custo, se a vagina produz um pH menor que quatro e meio pode diferenciar a candidíase de outras doenças como a Vaginose Bacteriana ou tricomoníase que elevam o pH (Duarte et al., 2019). 
Habitualmente, métodos bioquímicos são utilizados para identificação das espécies de leveduras do gênero Candida fundamentados em testes como a fermentação e assimilação de carboidratos, habilidade em formar tubo germinativo a $37^{\circ} \mathrm{C}$ em soro e produzir clamidoconídios em ágar fubá acrescido de Tween 80 (Menezes et al., 2012).

As condições predisponentes, a diversidade entre as leveduras, e a suscetibilidade de antifúngicos para $C$. albicans caracterizam a imunidade da mucosa vaginal como um fator-chave na patogênese da CVV recorrente a importância de realização de culturas para identificação das espécies de CNA, visando mais informações sobre essas leveduras (Dias et al., 2011). Desta forma, como abordado ao longo desta discussão é de suma importância a compreensão dos fatores moduladores, as características fisiológicas e os aspectos da microbiota vaginal.

\section{Conclusão}

De acordo com o exposto acima podemos evidenciar a importância da microbiota vaginal bem como, a interação do fungo Candida sp. com a microbiota, sua diversidade de espécie e a relação com os pacientes assintomáticos. O conhecimento dos sintomas, principalmente, durante a gravidez é uma importante ferramenta para o diagnóstico precoce durante o período prénatal. A transmissão vertical se mostra presente nas infecções em RN. A espécie mais evidente desse gênero é a $C$. albicans contudo, as espécies de CNA estão cada vez mais presentes nas infecções causadas pelo gênero Candida spp. Com isso, se faz necessário um diagnóstico objetivo e eficiente que ajudará no tratamento da infecção e melhorará o prognóstico do paciente.

Por fim, a avaliação da literatura permitiu avaliar e discutir os aspectos da microbiota vaginal e sua correlação com a candidíase vulvovaginal na gravidez, e suas formas de diagnóstico no cenário atual. No entanto, estudos anteriores já demonstravam complicações causadas pela candidíase na gestação, fazendo-se necessário a continuidade de estudos e a geração de novos dados para maior eficiência clínica no tratamento de Candidíase vulvovaginal na gestação. Sendo de grande valor novas pesquisas para maior consolidação das variações causadas pelo gênero Candida spp e o aumento de avaliações epidemiológicas para maiores compreensões clínicas.

\section{Referências}

Almeida, S. M. A., Bezerra, A. N., \& Mendonça, P. S. (2017). Efeito da suplementação de Lactobacillus spp. no tratamento e prevenção de candidíase vulvovaginal e vaginose bacteriana. Rev. Saúde Públ. Santa Cat., 10(3), 44-60 (2017).

Bernardo, K. M. R., \& Lima, A. P. W., (2015). Ocorrência de candidíase no exame citológico de pacientes do Hospital Geral de Curitiba. Revista Saúde e Desenvolvimento, 8 (4)

Dias, L. B., Melhem, M. S. C., Szeszs, M. W., Filho, J. M., \& Hahn, R. C. Vulvovaginal candidiasis in Mato Grosso, Brazil: Pregnancy status, causative species and drugs tests. Brazilian Journal of Microbiology (2011) 42: 1300-1307

Duarte, S. M. S., Faria, F. V., \& Martins, M. O., Métodos diagnósticos para a caracterização de candidíase e papilomavírus humano. Brazilian Journal of Development, 5 (10): 18083-18091

Freitas, L. F. Q., Maia, L. R. S., de Deus, M. R. A. R., Oliveira, S. R., \& Peres, A. L. Prevalência de microrganismos em secreção vaginal de gestantes de alto risco de uma maternidade em Caruaru, Pernambuco, Brasil. J Bras Patol Med Lab., 56: 1-6

Ghaddar, N., Anastasiadis, E., Halimeh, R., Ghaddar, A., Dhar, R., AlFouzan, W., Yusef, H., \& Chaar, M. E. Prevalence and antifungal susceptibility of Candida albicans causing vaginal discharge among pregnant women in Lebanon. BMC Infectious Diseases, 2020

Gonçalves, B., Ferreira, C., Alves, C. T., Henriques, M., Azeredo, J., \& Silva, S. Vulvovaginal candidiasis: Epidemiology, microbiology and risk factors. Crit Rev Microbiol, 2016; 42(6): 905-927

Lima, N. A., \& Cohen, J. V. F. B. (2017). Candidíase vulvovaginal recorrente em gestantes. Saber Científico.

Menezes, E. A., Júnior, A. A. V., Cunha, F. A., Cunha, M. C. S. O., Braz, B. H. L., Capelo, L. G., \& Silva, C. L. F. (2012).Identificação molecular e suscetibilidade antifúngica de Candida parapsilosis isoladas no Ceará, Brasil. J Bras Patol Med Lab. 48(6), 415-420.

Mucci, M. J., Cuestas, M. L., Landanburu, M. F., \& Mujica, M. T. (2017). Prevalence of Candida albicans, Candida dubliniensis and Candida africana in pregnant women suffering from vulvovaginal candidiasis.in Argentina. Rev Iberoam Micol. 34(2):72-76 
Research, Society and Development, v. 11, n. 1, e2911124704, 2022

(CC BY 4.0) | ISSN 2525-3409 | DOI: http://dx.doi.org/10.33448/rsd-v11i1.24704

Oliveira, J. A. G., \& Carneiro, C. M. Fatores associados a alterações da microbiota no trato genital feminino inferior. Pensar Acadêmico, Manhaçu, 18 (2): 289299

Peixoto, J. V., Rocha, M. G., Nascimento, R. T. L., Moreira, V. V., \& Kashiwabara, T. G. B. (2014). Candidíase - uma revisão de literatura. Brazilian Journal of Surgery and Clinical Research-BJSC, 8(2), 75-82.

Rodrigues, M. T., Gonçalves, A. C., Alvim, M. C. T., Filho, D. S. C., Zimmermmann, J. B., Silva, V. L., \& Diniz, C. G. (2013). Associação entre cultura de secreção vaginal, características sociodemográficas e manifestações clínicas de pacientes com diagnóstico de candidíase vulvovaginal. Rev Bras Ginecol Obstet. 35(12):554-61

Sangaré, I., Sirima, C., Bamba, S., Zida, A., Cissé, M., Bazié, W. W., Sanou, S., Dao, B., Menan, H., \& Guiguemdé, R. T. (2017). Prevalence of vulvovaginal candidiasis in pregnancy at three health centers in Burkina Faso. Journal De Mycologie Médicale.

Soares, D. M., Lima, E. O., Soares, D. M. M., Silva, N. F., Costa, N. G. M., Faria, F. S. E. D. V., \& Rodriguez, A. F. R. (2019). Candidíase vulvovaginal: uma revisão de literatura com abordagem para Candida albicans. Braz. J. Surg. Clin. Res. 25, 28-34

Souza, G. N., Vieira, T. C. S. B., Campos, A. A. S., Leite, A. P. L., \& Souza, E. (2012). Tratamento das vulvovaginites na gravidez. FEMINA. 40(3)

Tansarli, G. S., Skalidis, T., Legakis, N. J., \& Falagas, M. E. (2016). Abnormal vaginal flora in symptomatic non-pregnant and pregnant women in a Greek hospital: a prospective study. Eur J Clin Microbiol Infect Dis.

Tapia, C. V., Hermosilla, G., Fortes, P., Albuquenque C., Bucarey, S., Salinas, H., Rodas, P. I., Díaz, M. C., \& Magne, F. (2016).Genotyping and Persistence of Candida albicans from Pregnant Women with Vulvovaginal Candidiasis. Mycopathologia.

Vasconcelos, C. N. E., Silva, N. N. P., Batista, P. N., \& Kalil, J. H. (2016). Estudo comparativo entre terapia oral e local no tratamento de corrimentos vaginais: candidíase, tricomoníase e vaginose bacteriana. Brazilian Journal of Surgery and Clinical Research - BJSCR, 15(1), $123-128$.

Viera, A. J. H., \& Santos, J. I. (2017). Mecanismos de resistência de Candida albicans aos antifúngicos anfotericina B, fluconazol e caspofungina. RBAC. 49(3):235-9

Whibley, N., \& Gaffen, S. L. (2015). Beyond Candida albicans: Mechanisms of immunity to non-albicans Candida species. .

Zimmermmann, J. B., Santos, K. K., Alves, L. C., Vilano, L. S., Borges, N. A., Soares, J. P., Marangoni, M. C., Silveira, L. H. A., \& Nascimento, R. P. M. (2019). Frequência e etiologia de fluxos genitais na gravidez. Rev Fac Ciênc Méd Sorocaba. 21(2):65-8.

Zisova, L. G., Chokoeva, A. A., Amaliev, G., Ptleshkova, P. V., Liteva-Katrandzhieva, T. M., Krasteva, M. B., Uchikova, E. H., Kouz-manov, A. H., \& Ivanova, Z. V. (2016). Vulvovaginal Candidiasis in Pregnant Women and its Importance for Candida Colonization of Newborns. Folia Medica, 58(2) 